\title{
Appropriate tube size selection based on radiological images
}

\author{
Hee-Pyoung Park
}

Department of Anesthesiology and Pain Medicine, Seoul National University Hospital, Seoul National University College of Medicine, Seoul, Korea

When endotracheal intubation with a plain tube or doublelumen tube (DLT) is needed, selecting the appropriate tube size is important. A large tube may cause laryngotracheal injury, whereas a small tube may cause inadequate ventilation, due to air leak. Moreover, an overly large or small tube increases the chances of repeated intubation, which may cause airway trauma.

In clinical practice, selection of the tube size is usually based on clinical experience, which may be clinically acceptable in most adult patients. In pediatric patients, unfortunately, the age-based formula for predicting tube size, which is used worldwide, is not always successful because of differences in physical development [1]. Recently, technical developments in radiological imaging have allowed measuring the diameter of a patient's airway at various points. Chest computed tomography (CT) or plain X-ray can be used to predict the appropriate size of a DLT or an uncuffed plain tube by measuring the diameter of the left main bronchus or the tracheal transverse diameter at the $7^{\text {th }}$ cervical vertebra [1-5]. Moreover, because the tracheal width is closely correlated with the left main bronchus width, if direct measurement of the left main bronchus width is impossible, the tracheal width can be more helpful in estimating the left main bronchus width than demographic data, such as height, weight, and age $[6,7]$.

Ultrasound can also be useful for measuring the diameter of a patient's airway, especially the tracheal diameter. Sustić and colleagues demonstrated a strong correlation between the tracheal width measured by ultrasound and the tracheal width by
CT [8]. Additionally, they found a strong correlation between the tracheal width, measured by ultrasound, and the left main bronchus width, measured by CT, and that $25 \%$ of patients had the incorrect size of left-sided DLT. In pediatric patients, ultrasound can also be helpful in selecting the proper size of an uncuffed endotracheal tube [9].

More recently, three-dimensional (3-D) spiral chest CT, with which multi-planar reconstruction is possible, has been introduced. It allows more precise evaluation of a patient's airway. A study in this issue of the Korean Journal of Anesthesiology [10] demonstrated a moderate correlation between the anteroposterior (AP) diameter at $2 \mathrm{~cm}$ below the carina of the left main bronchus, obtained from the 3-D images, and the 2-D images, and that the AP diameter was longer on 3-D images than on 2-D images. In the study, if left-sided DLTs were chosen based on the AP diameter measured $2 \mathrm{~cm}$ below the carina of the left main bronchus from the 3-D and 2-D images, the same sized leftsided DLTs were expected in $51 \%$ of patients. This suggests that prediction of the left-sided DLT size, based on the AP diameter on 2-D images, may be reconsidered, although a large prospective study should be performed to confirm the superiority of 3-D images in predicting the appropriate size of left-sided DLT.

In conclusion, appropriate tube size selection is important. Tube size selection based on radiological images may be reasonable and may have a greater likelihood of successful intubation at the first attempt than tube size selection based on clinical experience.

Corresponding author: Hee-Pyoung Park, M.D., Ph.D., Department of Anesthesiology and Pain Medicine, Seoul National University Hospital, Seoul National University College of Medicine, 101, Daehak-ro, Jongno-gu, Seoul 110-744, Korea. Tel: 82-2-2072-2466, Fax: 82-2-747-5639, E-mail: hppark@snu.ac.kr

() This is an open-access article distributed under the terms of the Creative Commons Attribution Non-Commercial License (http:// creativecommons.org/licenses/by-nc/3.0/), which permits unrestricted non-commercial use, distribution, and reproduction in any medium, provided the original work is properly cited. 


\section{References}

1. Park HP, Hwang JW, Lee JH, Nahm FS, Park SH, Oh AY, et al. Predicting the appropriate uncuffed endotracheal tube size for children: a radiograph-based formula versus two age-based formulas. J Clin Anesth 2013; 25: 384-7.

2. Chow MY, Liam BL, Thng CH, Chong BK. Predicting the size of a double-lumen endobronchial tube using computed tomographic scan measurements of the left main bronchus diameter. Anesth Analg 1999; 88: 302-5.

3. Eberle B, Weiler N, Vogel N, Kauczor HU, Heinrichs W. Computed tomography-based tracheobronchial image reconstruction allows selection of the individually appropriate double-lumen tube size. J Cardiothorac Vasc Anesth 1999; 13: 532-7.

4. Hannallah MS, Benumof JL, Ruttimann UE. The relationship between left mainstem bronchial diameter and patient size. J Cardiothorac Vasc Anesth 1995; 9: 119-21.

5. Jeon Y, Ryu HG, Bahk JH, Jung CW, Goo JM. A new technique to determine the size of double-lumen endobronchial tubes by the two perpendicularly measured bronchial diameters. Anaesth Intensive Care 2005; 33: 59-63.

6. Brodsky JB, Lemmens HJ. Tracheal width and left double-lumen tube size: a formula to estimate left-bronchial width. J Clin Anesth 2005; 17: 267-70.

7. Brodsky JB, Malott K, Angst M, Fitzmaurice BG, Kee SP, Logan L. The relationship between tracheal width and left bronchial width: Implications for left-sided double-lumen tube selection. J Cardiothorac Vasc Anesth 2001; 15: 216-7.

8. Sustić A, Miletić D, Protić A, Ivancić A, Cicvarić T. Can ultrasound be useful for predicting the size of a left double-lumen bronchial tube? Tracheal width as measured by ultrasonography versus computed tomography. J Clin Anesth 2008; 20: 247-52.

9. Bae JY, Byon HJ, Han SS, Kim HS, Kim JT. Usefulness of ultrasound for selecting a correctly sized uncuffed tracheal tube for paediatric patients. Anaesthesia 2011; 66: 994-8.

10. Lee JW, Son JS, Choi JW, Han YJ, Lee JR. The comparison of the lengths and diameters of main bronchi measured from two-dimensional and three-dimensional images in the same patients. Korean J Anesthsiol 2014; 66: 189-94. 